Session 3464

\title{
Teaching About Materials Using Electronic Devices
}

\author{
Sarah E. Leach \\ Purdue University
}

\begin{abstract}
Introductory materials courses must, of necessity, contain a great deal of theoretical and foundational information about the structure and properties of materials. Material categories are often studied separately, with comparisons being made between types. This paper describes a laboratory experience designed to bring together different types of materials, by studying complex manufactured devices. Many electronic packaging systems, including chip carriers and thick-film circuits, comprise many layers of various material types. The polymer, ceramic, and metallic materials used to construct and package electronic devices are chosen and combined carefully to take advantage of the distinctive physical and mechanical properties of each material category. During the lab session students dissect and examine devices and electronic packaging systems to understand more about the ways materials can be used together to create complex structures. The lab was designed for lower division students who are not materials science majors. Emphasis is placed on understanding the physical and mechanical properties involved in material selection, and on understanding how combinations of material types can be used to satisfy design requirements.

Background

This laboratory exercise is presented near the end of the semester, after students have learned about material structure, the relationships between structures and both physical and mechanical properties, the concepts of stress and strain, and the general characteristics of different families or types of materials. Application examples utilized to this point in the semester are usually large, common objects, for example; automobiles, bicycles, airplanes, toasters, hammers, skis, or golf clubs. This laboratory exercise does not introduce entirely new materials or concepts, but serves to reinforce these concepts using very small functional structures and the new combinations of materials.
\end{abstract}

\section{Description}

A brief lecture introduces the students to the history of electronic packaging, beginning with examples of macroscopic wiring like a lamp or a simple circuit on a breadboard. The technological transformation of electrical and electronic applications from soldered wires to printed circuit boards to devices carried on silicon chips is illustrated with physical samples, photographs, and packaging industry literature. Emphasis is placed on the idea that packaging applications have become smaller and smaller, while simultaneously having increased functionality and becoming less expensive. ${ }^{1,2}$ Students are introduced to a brief glossary of terms and acronyms, to give them an understanding of some of the nomenclature and typical configurations used in packaging applications. Listed below is an example of a glossary to be distributed to students. 
An Abbreviated Glossary for Electronic Packaging:

Component - a functional part of an electronic circuit. Can be discrete, for example; a resistor, a capacitor, a switch, or a connector. Components can also be integrated into devices. (e.g. integrated circuits)

Device - a functional unit which can be considered a self-contained and made up of components. Examples: a CD player, an amplifier, or a clock (circuit).

Chip - circuitry on a (usually) silicon wafer, cut up into individual functional units (or devices).

Wafer - a thin disk of silicon, used in making chips

Solder Ball - just what it sounds like. A very small ball of solder, used to attach a chip or device to a substrate.

Ball Grid Array - an array of solder balls, used to connect (physically and electrically) many locations simultaneously.

Substrate - a physically robust carrier or platform. Used to hold, protect, and provide electrical connections to chips or small devices.

During the final segment of the introduction, students are shown a (non-functional) floppy disc drive from a personal computer. A review is given of the basic function of the drive, and the component parts one would expect to find inside. Special consideration is given to the read/write head and accompanying circuitry, which must satisfy potentially competing requirements of small spacing, multiple electrical paths, low mass, and stringent dimensional stability. Figure 1 shows an example of an assembly, composed of polymeric, ceramic, and metallic materials. This example also shows a flexible circuit cable constructed with copper traces on a polyimide substrate. ${ }^{3}$

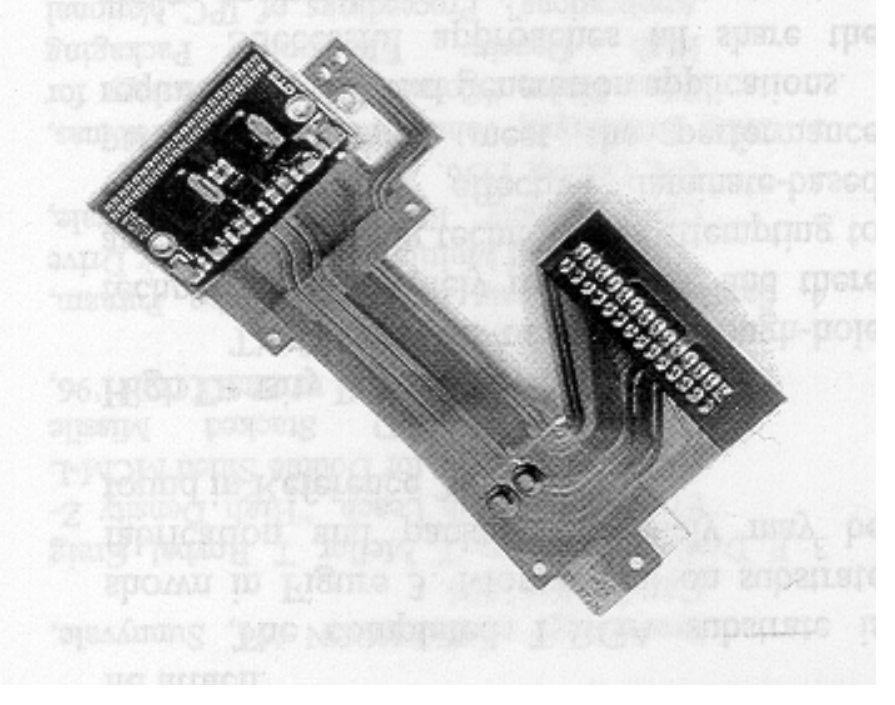

Figure 1 - Floppy Drive Read/Write Circuitry and Cable

After this introduction, students are assigned to teams and instructed to work for approximately 15 minutes at each of three stations, rotating through the stations until 
each group has worked at each station. At each station the team is directed to read and answer a series of questions. The first station consists of a series of papers, packaging industry publications, and technology information data sheets. The team is directed to scan through the printed material and gain some sense of the kinds of materials issues that are important to electronic packaging. They are also expected to develop a sense of the technical background necessary to work on packaging projects or research. A list of internet-based references is also provided to assist them in locating further information.

The second experimental station is set up for microscopic examination of some devices and packages. Low power (10-40x) optical microscopes are available, as are a collection of hybrid circuits, printed circuit boards, and chip carriers, both complete and crosssectioned and mounted in transparent epoxy. Clock oscillators, which are hybrid devices constructed from ceramic substrates, with quartz crystal oscillators mounted on metallic supports and screen-printed circuit components, all protected beneath a metal "lid" are good examples of complex arrangements of materials used together. With additional kinds of samples, this station also allows the students to examine wire bonds, buried conductive traces, patterned silicon chips, solder ball attachment arrays, and other structures. Printed diagrams explain what materials are present in the various devices.

The third experimental station is prepared so that the students can disassemble a floppy disc drive and make observations about the design of the device as it relates to the materials used. Students are asked to record the components they "discover" and describe the material or materials they find. References are also given to other uses or recycling requirements for outdated electronic equipment. ${ }^{4}$

To complete the lab assignment, each student team is expected to submit a written report containing the answers to the assigned questions from each station. Students are encouraged to use their textbook and supplemental references to make comparisons about material properties. In order to guide the students and enhance their learning experience, questions are posed based on Bloom's Taxonomy of Educational Objectives. ${ }^{5}$ Typical questions are listed below, along with the relevant work station location(s):

1. (third work station)What physical and mechanical properties do you think might be important for electronic packaging, and why? Describe at least five parts of the floppy drive read/write assembly and explain why the materials used were chosen.

2. (second work station) What other methods might you suggest for attaching or connecting devices or components in electronic packages?

3. (first and second work stations) What considerations other than material properties might be important for electronic packaging design?

4. (first and second work stations) What mechanical and physical properties are desirable for the wire used in wire bonding?

5. (first work station) Choose one of the technical papers in the folder and write a short paragraph explaining the topic of the paper and its major finding or result.

The first, fourth, and fifth questions require that the students have mastery or knowledge of earlier material in the course, and that they comprehend the requirements of electronic packaging applications. The second question attempts to determine whether the students 
can analyze and synthesize or generalize using what they understand about manufactured products and the electronics industry. The third question asks them to demonstrate that they can apply what they know and understand to suggest new solutions. This selection of questions is not intended to be limiting, but to suggest a set of directed inquiries which can help develop student learning and provide an assessment tool for the instructor. The written report format also provides an opportunity for students to demonstrate their written communication skills.

Assessment

The laboratory assignment has only been offered for one group of twenty-five students, so the report questions and assessment rubrics are still in their formative stages. Based on responses from the first group of students, a few generalizations can be made. Almost all of the students were able to demonstrate a clear understanding of the physical structure and function of several kinds of electronic packaging configurations. Students were able to demonstrate mastery of earlier course material by relating material properties to packaging requirements. (Questions 1 and 4.) All of the students offered connection alternatives as required in question 2 and material considerations for question 3,but it is not clear how many of the responses were based on independent thought or synthesis as opposed to responses based on research outside the classroom. A modified question asking the student to find alternatives based on research and then giving the student the option to list additional, original ideas would help distinguish between the learning objectives of research/knowledge and application/synthesis. Based on the assessment todate, it seems to be advantageous to provide students with only basic information including a glossary, descriptions of some basic packaging configurations, and a list of suggested references, and then allow them to "discover" other configurations and material uses.

Summary

Electronic packaging applications can provide an accessible, inexpensive vehicle for introducing students to complex structures. Floppy disk drives and other electronic devices or assemblies are typically available at low-cost or no-cost and so make ideal samples for student dissection and analysis. Directed questions can also enhance the learning experience for students by requiring that they bring together their mastery of previous course information, their ability to grasp new meanings situations, and apply their knowledge and comprehension to solve new problems. The technological change demonstrated in the design of electronic packaging systems is also a good illustration of the rapid pace of change in manufacturing practice and materials selection.

Bibliography

1. Gilleo, K., "Flex Takes over the World...Maybe!" in Proceedings of the Third International Conference on Flex Circuits, October 23-25, Sunnyvale, CA 1996 pp. 1-8.

2. Harper, C.A., Ed., Electronic Packaging and Interconnect Handbook, McGraw-Hill, 1991

Proceedings of the 2004 American Society for Engineering Education Annual Conference \& Exposition Copyright $\left({ }^{C}\right.$ 2004, American Society for Engineering Education 
3. Leach, S.E. and Ernsberger, C.N., "Colamination Technology for Electronic Packaging Applications" in Proceedings of the Third International Symposium and Exhibition on Advanced Packaging Materials, Braselton, GA March 9-12, 1997 pp. 38-41

4. Hrynkiw, D. and Tilden, M.W., Junkbots, Bugbots \& Bots on Wheels, McGrawHill/Osborne, Berkely, CA 2002

5. Bloom, B.S. and Krathwohl, D.R. "Taxonomy of Educational Objectives: The Classificaiton of Educational Goals, by a committee of college and university examiners". Handbook I: Cognitive Domain. New York, Longmans, Green, 1956.

Internet Resources

http://extra.ivf.se/ngl/ The Nordic Electronics Packaging Guideline webpage. This site has very good introductory information about electronic packaging configurations with photographs and schematics.

http://www.packaging.buffalo.edu/ SUNY Buffalo Electronic Packaging Lab. http://nepp.nasa.gov/index nasa.cfm/778/ NASA Electronic Parts and Packaging Program. http://www.dcsimtech.com/code/ep-00.html Daimler-Chrysler SIM Electronic Packaging. http://www.ieec.binghamton.edu/ieec/ SUNY Binghamton Integrated Electronics Engineering Center. http://www.prc.gatech.edu/ Georgia Tech Microsystems Packaging Research Center. http://www.calce.umd.edu/general/bga work.htm Computer Aided Life Cycle Engineering (CALCE) Electronic Products and Systems Center at the University of Maryland, research on ball grid array packaging. http://nanotron.ecn.purdue.edu/Research/etsim.htm List of research being done on thermal management of electronic devices at the Purdue University Nanoscale ThermoFluids Laboratory. http://www.national.com/packaging/ National Semiconductor's Packaging site, contains application notes for various package configurations.

Sources for Parts and Devices

www.digikey.com 1-800-344-4539

www.allelectronics.com $\quad 1-800-826-5432$

Biography

SARAH LEACH is an Assistant Professor in the Mechanical Engineering Technology department of the Purdue University, School of Technology. Her primary teaching responsibilities are in materials and applied mechanics. 